\title{
High-Average-Power Diode-Pumped Yb:YAG Lasers
}

R. J. Beach, E. C. Honea, S. B. Sutton, C. M. Bibeau, J. A. Skidmore, M. A. Emanuel, S. A. Payne, P. V. Avizonis, R. S. Monroe, D. G. Harris

This article was submitted to International Forum on Advanced High-Power Lasers and Applications, Suita, Japan, November 1-5, 1999

U.S. Department of Energy

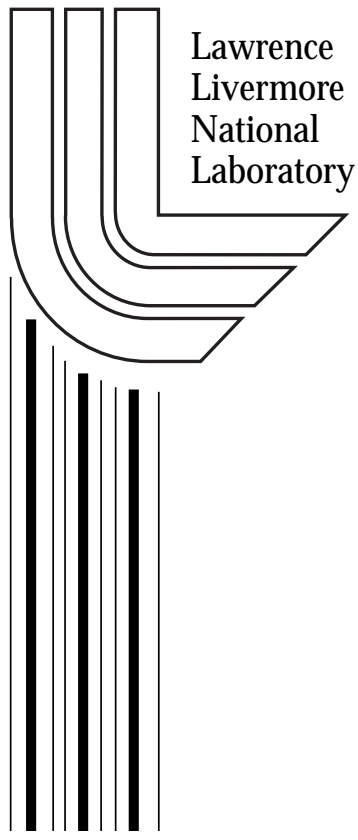

\section{October 1, 1999}




\section{DISCLAIMER}

This document was prepared as an account of work sponsored by an agency of the United States Government. Neither the United States Government nor the University of California nor any of their employees, makes any warranty, express or implied, or assumes any legal liability or responsibility for the accuracy, completeness, or usefulness of any information, apparatus, product, or process disclosed, or represents that its use would not infringe privately owned rights. Reference herein to any specific commercial product, process, or service by trade name, trademark, manufacturer, or otherwise, does not necessarily constitute or imply its endorsement, recommendation, or favoring by the United States Government or the University of California. The views and opinions of authors expressed herein do not necessarily state or reflect those of the United States Government or the University of California, and shall not be used for advertising or product endorsement purposes.

This is a preprint of a paper intended for publication in a journal or proceedings. Since changes may be made before publication, this preprint is made available with the understanding that it will not be cited or reproduced without the permission of the author.

This report has been reproduced directly from the best available copy.

Available to DOE and DOE contractors from the

Office of Scientific and Technical Information

P.O. Box 62, Oak Ridge, TN 37831

Prices available from (423) 576-8401

http:/ / apollo.osti.gov/bridge/

Available to the public from the National Technical Information Service

U.S. Department of Commerce 5285 Port Royal Rd., Springfield, VA 22161 http://www.ntis.gov/

OR

Lawrence Livermore National Laboratory Technical Information Department's Digital Library http://www.llnl.gov/tid/Library.html 


\title{
High-Average-Power Diode-Pumped Yb:YAG Lasers
}

\author{
R.J. Beach, E.C. Honea, S.B. Sutton, C.M. Bibeau, J.A. Skidmore, M.A. Emanuel, and S.A. Payne \\ Lawrence Livermore National Laboratory, L-441, P.O. Box 808, Livermore, California 94551 \\ P.V. Avizonis, R.S. Monroe, and D.G. Harris \\ Boeing Information, Space \& Defense Systems, MS SZ-15, 2800 Westminster Boulevard, Seal Beach, \\ California 90740
}

\begin{abstract}
A scaleable diode end-pumping technology for high-average-power slab and rod lasers has been under development for the past several years at Lawrence Livermore National Laboratory (LLNL). This technology has particular application to high average power Yb:YAG lasers that utilize a rod configured gain element. Previously, this rod configured approach has achieved average output powers in a single 5 $\mathrm{cm}$ long by $2 \mathrm{~mm}$ diameter $\mathrm{Yb}$ :YAG rod of $430 \mathrm{~W} \mathrm{cw}$ and $280 \mathrm{~W}$ q-switched. High beam quality $\left(\mathrm{M}^{2}=2.4\right)$ $\mathrm{q}$-switched operation has also been demonstrated at over $180 \mathrm{~W}$ of average output power ${ }^{1}$. More recently, using a dual rod configuration consisting of two, $5 \mathrm{~cm}$ long by $2 \mathrm{~mm}$ diameter laser rods with birefringence compensation, we have achieved $1080 \mathrm{~W}$ of $\mathrm{cw}$ output with an $\mathrm{M}^{2}$ value of 13.5 at an optical-to-optical conversion efficiency of $27.5 \%{ }^{2}$. With the same dual rod laser operated in a q-switched mode, we have also demonstrated $532 \mathrm{~W}$ of average power with an $\mathrm{M}^{2}<2.5$ at $17 \%$ optical-to-optical conversion efficiency. These q-switched results were obtained at a $10 \mathrm{kHz}$ repetition rate and resulted in $77 \mathrm{nsec}$ pulse durations. These improved levels of operational performance have been achieved as a result of technology advancements made in several areas that will be covered in this manuscript. These enhancements to our architecture include: (1) Hollow lens ducts that enable the use of advanced cavity architectures permitting birefringence compensation and the ability to run in large aperture-filling near-diffraction-limited modes. (2) Compound laser rods with flanged-nonabsorbing-endcaps fabricated by diffusion bonding. (3) Techniques for suppressing amplified spontaneous emission (ASE) and parasitics in the polished barrel rods.
\end{abstract}

\section{Introduction}

The impact that semiconductor lasers have had on the development of solid-state lasers is evident in highaverage-power solid-state laser systems, which are finding wide application in the field of materials processing. Commonly referred to as HAP DPSSLs, for high-average-power diode-pumped solid-state lasers, these systems are best characterized as being performance limited by thermal management issues associated with the solid state laser crystal. These thermal management issues can manifest themselves in several different forms, such as aberration of beam quality due to thermally induced refractive index variations in the crystal, and in extreme cases, fracture of the laser crystal due to the buildup of excessive stress in the crystal. The most common ion / host crystal combination used in HAP DPSSL systems today is $\mathrm{Nd}^{3+}$ :YAG. Of all the well-developed laser crystals that can be grown with rare-earth impurities, YAG is the most robust in a thermal sense; i.e., it has the highest thermal conductivity and the highest resistance to fracture. As such, it is natural that YAG is the crystalline host of choice for HAP DPSSLs. The wide use of $\mathrm{Nd}^{3+}$ as the active ion in nearly all HAP DPSSLs is associated with several fortuitous coincidences between the pump irradiance and the wavelength required to efficiently excite it, and the pump irradiance and the wavelength that can be conveniently developed by two-dimensional laser diode arrays.

Although the $\mathrm{Nd}^{3+}$ ion presently dominates the HAP DPSSL field, the situation could change in the future, due to continued laser diode array technology developments ${ }^{3,4}$. One of these developments, which has been actively pursued at LLNL, is a diode array pump technology that enables pump power to be delivered at much higher irradiances than was previously possible ${ }^{5}$. Because many rare-earth ion based HAP DPSSLs are only practical if pumped at higher irradiances than the several $\mathrm{kW} / \mathrm{cm}^{2}$ that is possible with conventional scaled diode array technologies, this approach has opened a path to HAP DPSSLs that rely on ions other than $\mathrm{Nd}^{3+}$ as their lasant. We have demonstrated a HAP Yb:YAG DPSSL which presently leads the world for average brightness generation in its class, as discussed in detail below. 


\section{Single rod Yb:YAG end-pumped laser}

Figure 1 shows a schematic drawing of our single-rod Yb:YAG laser that serves to illustrate the endpumping technology that we have developed along with other recent improvements that we have made. The laser diode pump array consists of a stack of 36 average power silicon microchannel cooled laser diode packages ${ }^{6}$. Each of these packages holds a $1.5 \mathrm{~cm}$ long laser diode bar that has its output radiation collected and collimated by a high performance shaped-fiber cylindrical-microlens ${ }^{7,8}$. The diode stack consists of two separate sub-stacks, each one containing 18 packages, which are separated in the middle by

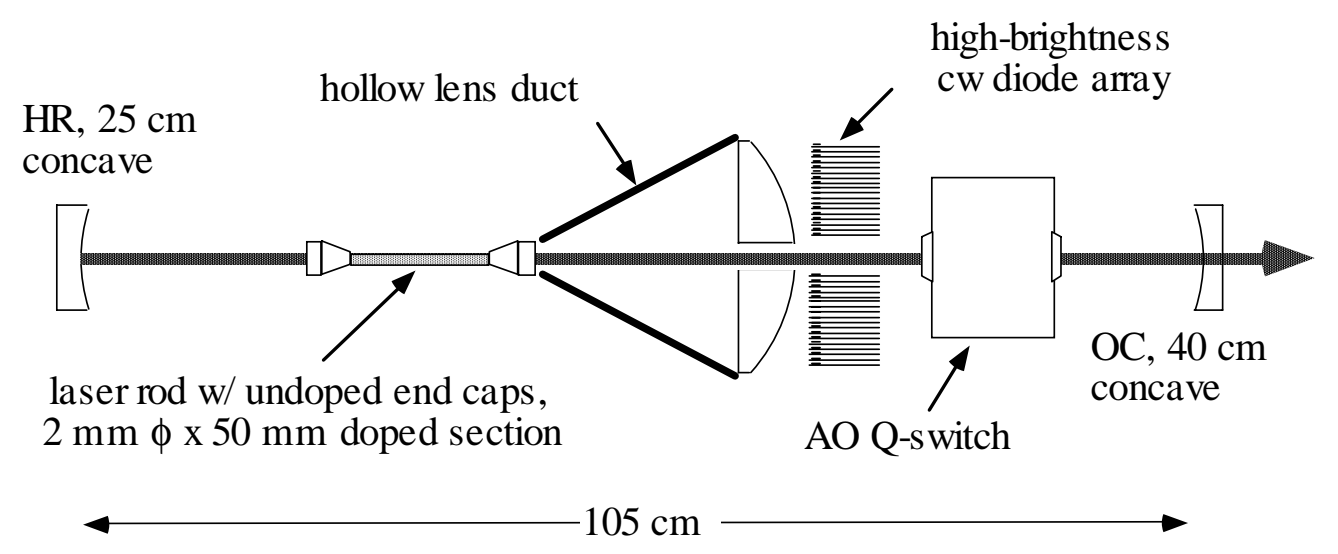

Fig. 1. Yb:YAG end-pumping architecture using microlens-conditioned laser diode arrays and a hollow lens duct to deliver the laser diode pump radiation into a composite laser rod.

a spacer with a $6 \mathrm{~mm}$ hole in it to allow the laser beam to pass through the hollow lens duct (HLD) unobstructed. This laser has recently produced $452 \mathrm{~W}$ of cw output at an optical-optical efficiency of $19.3 \%$. This level of performance was obtained with a measured beam quality $\left(\mathrm{M}^{2}\right)$ of 3.7 by designing the resonator to have a large transverse fundamental mode, as described in reference [1]. What follows is a description of the component technologies that have enabled this level of performance.

\section{Hollow lens duct}

Figure 2 shows a sketch of the HLD that we have developed for laser applications in which it is important to have access to the laser beam at both ends of the laser rod. Previous to the development of the hollow lens duct, we have used solid lens ducts fabricated from transparent optical materials such as fused silica.

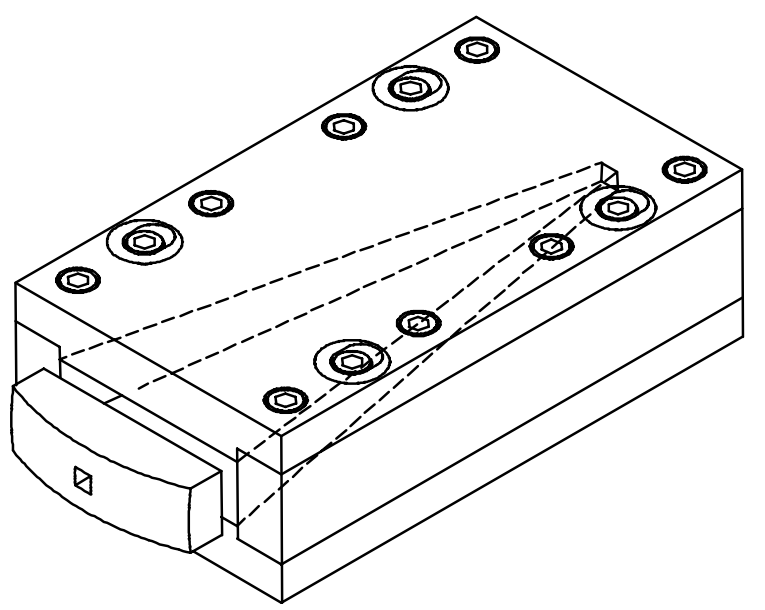

Fig. 2. Schematic drawing of hollow lens duct. The spherical lens at the pump input end of the duct has a hole fabricated in it to allow the laser beam to exit the assembly unobstructed. 
These solid devices worked by lensing at their curved input surface and then ducting by total internal reflection (TIR) at their canted planar sides ${ }^{9}$. One limitation of the solid lens duct pump delivery architecture is that it requires a dichroic coating on the pump input end of the laser rod, which serves to reflect the laser light but transmit the pump light, limiting the approach to cavities with a high reflector on one end of the laser $\operatorname{rod}^{10,11}$. In contrast, the hollow lens duct allows more flexible cavity designs, as shown in Fig. 1, where the laser rod can be located at an arbitrary position within the laser cavity. Our HLDs are fabricated from four pieces of aluminum that fit together as shown in Fig. 2, and have a protected-silver coating on their inner planar surfaces, which serve to reflect the pump light. The lens at the pump input end of the duct is a commercially procured plano-convex spherical lens that we cut to the shape shown and then mill a hole into. The milled hole in the lens is situated on the same axis as the hole in the center of the laser diode array, allowing the laser beam to clear the structure unobstructed. The back focal length of the lens is chosen so that the pump radiation will come to a focus in the laser rod and is typically slightly longer than the axial length of the HLD.

The most important performance metric of a hollow lens duct is the transfer efficiency for transferring pump light from the diode array into the laser rod. Figure 3 plots the diode pump power measured both

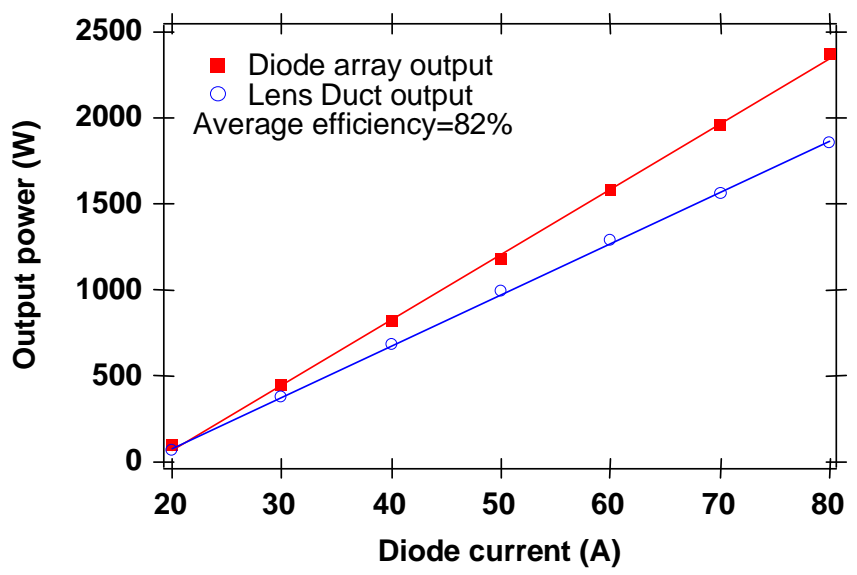

Fig. 3. The measured transfer efficiency of the hollow lens duct is $82 \%$.

before and after the HLD used with the laser shown in Fig. 1. This particular hollow lens duct collects light from a diode array with outside dimensions of $5 \mathrm{~cm} \times 1.5 \mathrm{~cm}$ and delivers it through a rectangular end opening measuring $4.6 \mathrm{~mm} \times 2.6 \mathrm{~mm}$ on a side, for a compression ratio of 63, with a transfer efficiency of 0.82. The same method of images construction that is useful for designing and optimizing solid lens ducts as explained in reference [9], is also applicable to the presently considered HLDs. Figure 4 shows this method of images construction that results in a torus surface being traced out by the lens duct output face when the lens duct is repeatedly reflected about its canted planar sides. The photograph on the right side of Fig. 4 is taken looking down into the HLD from the pump input end, and clearly shows the faceted torus surface formed by HLD output aperture under multiple reflections. In addition to the method of images analysis described in reference [9], we also have performed ray trace modeling, starting with the measured angular distribution of the microlens conditioned light at the laser diode array. In this analysis, a montecarlo ray trace is performed tracking emitted rays of pump light from the microlens-conditioned diode package, through the spherical lens on the input face of the duct, and then through the reflections made in traversing the hollow structure. For the presently considered hollow lens duct along with its $5 \mathrm{~cm} \mathrm{x} 1.5 \mathrm{~cm}$ diode array, this analysis is shown in Fig. 5. One of the very useful features of this ray trace analysis is that it yields the angular divergence of the pump radiation on exiting the hollow duct, which then allows the pump light to be traced into and through the laser rod. 

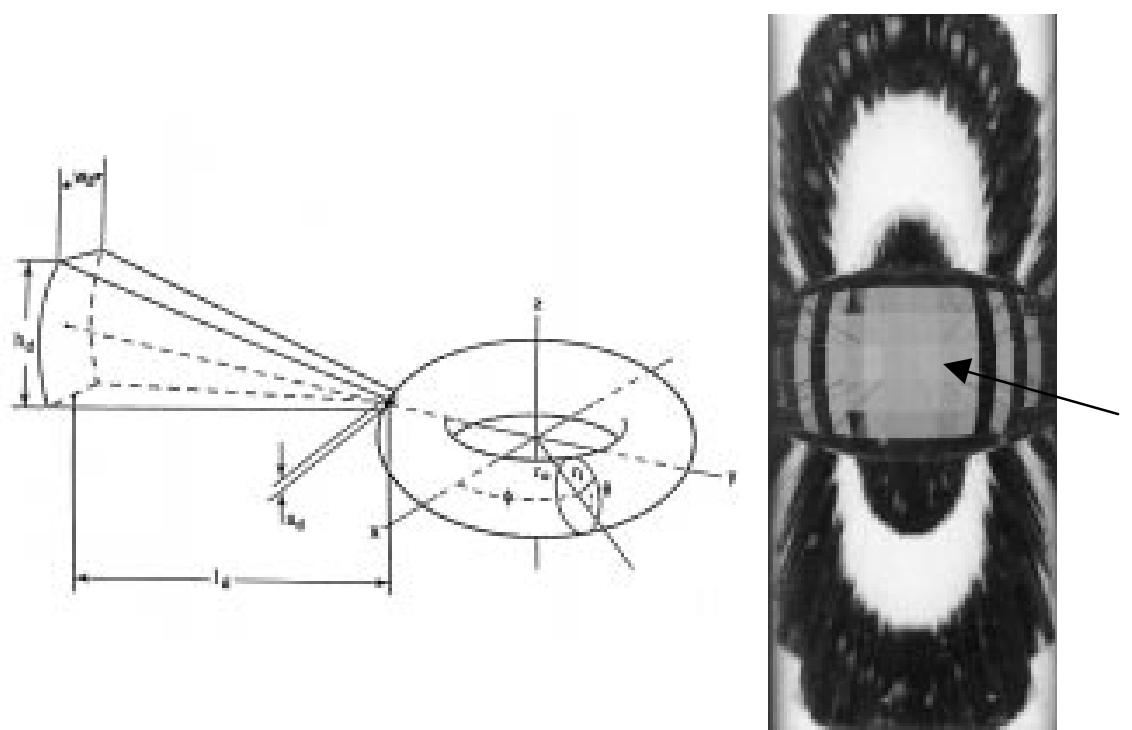

faceted

torus

surface

Fig. 4. The drawing illustrates method of images construction that results in the output face of the lens duct tracing out a torus surface. This construction simplifies the design and optimization of hollow lens ducts. The photograph on the right is taken looking down the hollow lens duct and clearly shows the faceted torus surface that is generated by the multiple reflections off the inside walls of the structure.

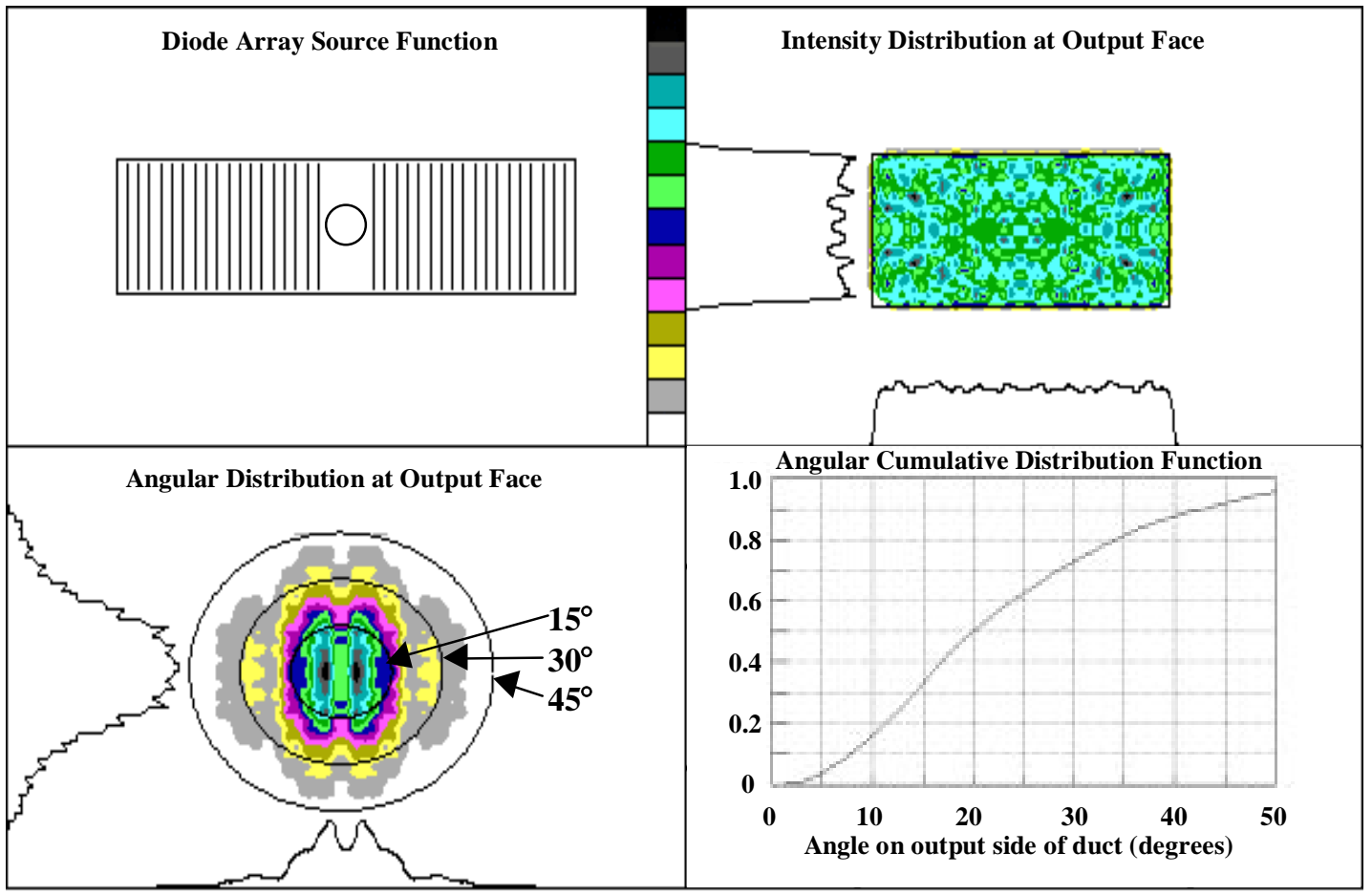

Fig. 5. Ray trace analysis of lens duct. Clockwise from the top left: (1) The diode array layout showing the split between the two sub-stacks and the hole in the center spacer for the laser beam to exit through the duct and array unobstructed. (2) The intensity distribution of the pump radiation at the rectangular output aperture of the hollow lens duct. (3) The angular distribution of the pump radiation at the hollow lens duct output aperture. (4) The angular cumulative distribution function for the radiation at the hollow lens duct output aperture. 


\section{Laser rods}

One of the critical developments that have made the end-pumping technology successful has been the development of laser rods that incorporate undoped endcaps ${ }^{12}$. Figure 6 depicts two laser rods that are being end-pumped, one with an undoped endcap and one without. Focussing on the top rod in Fig. 6 (the rod without an endcap), the surface of the pump input face tends to bulge out under thermal load due the thermal expansion of the rod material under heat load. This bulging means that the pump input surface is in tensile strain and therefore at risk of fracture. In fact, fracture of the pump-input surface was one of the early problems encountered in our development of the end-pumped laser technology. Our solution to this problem was to incorporate an undoped endcap onto the end of the laser rod that is attached to the doped portion of the rod through a diffusion bonding process that has been commercialized by ONYX Optics Corporation $^{13}$. Because the diffusion bond strength approaches the strength of the bulk material, the effect of the undoped endcap is to keep the pump input face on the doped portion of the laser rod buried in the bulk material. Thus, this surface which is in tensile strain without an endcap is now held in compressive strain, eliminating the risk of fracture.

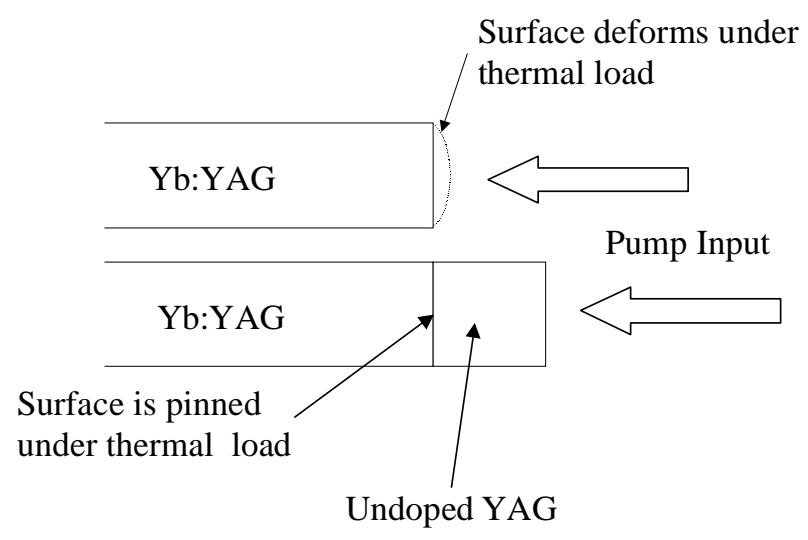

Fig. 6. One of the initial problems encountered in the LLNL developed end-pumping technology was the fracture of the pump-input face of the laser rod. Utilizing laser rods with undoped endcaps is an effective way to keep the thermally loaded pump input surface of the doped portion of the laser rod pinned in compression, thereby avoiding fracture of the pumped end-face of the rod.

An additional benefit of the endcap is that it isolates somewhat the optical coating on the end of the laser rod from the thermally loaded portion of the rod. Particularly in cases where a dichroic coating is required on the pump input end of a laser rod, this aspect of the endcap is beneficial as such coatings often exhibit a temperature sensitivity that can degrade their performance. The temperature plots shown in Fig. 7 illustrate this point by comparing an ordinary laser rod without an endcap, to laser rods with undoped endcaps under conditions of similar thermal loading.

The bottom laser rod in Fig. 7 depicts one of the enhancements that have been made to endcaps since their original introduction. The use of a flanged endcap on the end of the laser rod effectively moves the o-rings that are used to seal the rod into its water cooled head farther away from the low angle scatter of the laser beam than if no flange were used. This was found to be an important enhancement for robust highaverage-power operation of our end-pumped Yb:YAG laser systems, as we observed o-ring degradation when using unflanged rods. Another benefit of the flanged endcap was found to be its effectiveness at suppressing ASE and parasitic lasing in the polished barrel rods that are used in our end-pumping approach, ${ }^{11}$ as will be discussed in more detail in the next section. 


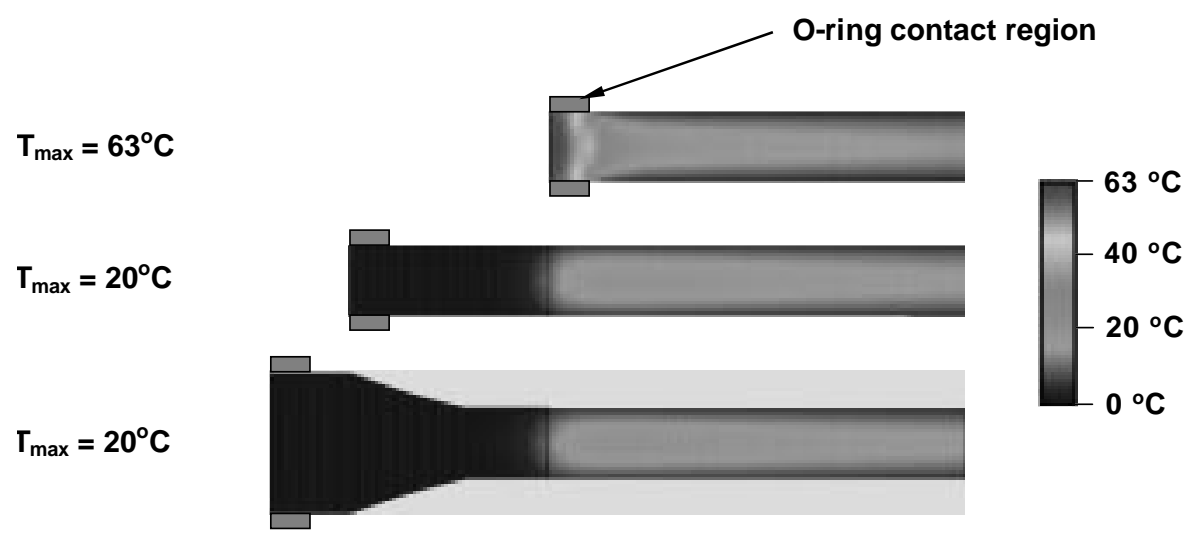

Fig. 7. Temperature profiles of three similarly loaded laser rods. From top to bottom are shown a laser rod without an undoped endcap, a laser rod with a conventional undoped endcap, and a laser rod with a flanged undoped endcap.

The most recent enhancement that has been made to our endcap technology is the development of endcaps with a rectangular cross-section. Previously, all flanged endcaps that were used in our laser systems were cylindrically symmetric, i.e., round cross-sections. However, we have found that we can increase our pump light transfer efficiency from the diode array into the laser rod by using endcaps with rectangular crosssection that smoothly transition to the round laser rod as illustrated in Fig. 8. The rectangular endcaps present a better impedance match to the diode light from the lens duct to the laser rod and are designed so

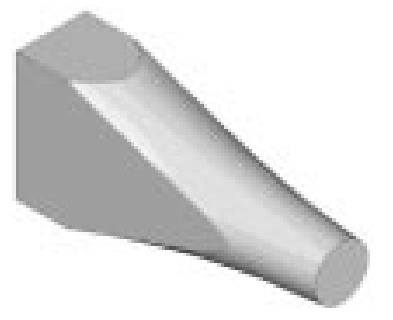

Fig. 8. Rectangular flanged endcap on laser rod serves as a continuation of the ducted pump delivery system.

that they look just like a continuation of the lens duct to the pump light. This is the reason that the back focal length of the plano-convex lens used at the input surface of the hollow lens duct typically has a slightly longer focal length than the axial length of the hollow lens duct. Calling $\ell_{\mathrm{HD}}$, the length of the hollow lens duct and $\ell_{\mathrm{F}}$, the length of the flanged rod end, the back focal length, $\mathrm{F}$, of the plano-convex lens on the pump input end of the hollow lens duct is optimized by,

$$
F=\ell_{H D}+\frac{\ell_{F}}{n}
$$

where $\mathrm{n}$ is the refractive index of the material that the flange is fabricated from, which in the presently considered case is YAG. 


\section{ASE and Parasitic Supression}

Another issue that the end-pumping technology developed at LLNL initially had, arose from parasitics and ASE, problems that were exacerbated by the polished barrel rods that were used to confine and duct pump light down their length by TIR. The same polished barrels used to duct the pump light also efficiently support and promote barrel modes. It is interesting to note that in our earliest lasers that used the end-pumping technology, which were $2 \mu \mathrm{m}$ Tm:YAG lasers, this problem was not noticed, presumably because the barrel modes in the Tm:YAG laser rods would quench due to $2 \mu \mathrm{m}$ absorption of the barrel mode's evanescent wave in the cooling water which surrounds the laser rod. As early as 1965 there have been reports in the literature regarding the impact of trapped light in polished barrel laser rods $^{14}$. The established picture of trapped laser light is shown in Fig. 9. Trapped by TIR, light can swirl around the inside of the laser rod and travel over long path lengths before reaching the end of the rod. The result of these long trapped ASE paths is that the gain present in the outer portion of the laser rod can be effectively depleted. If $n_{r}$ is the refractive index of the laser rod, and $n_{s}$ is the refractive index of the material surrounding the laser rod, then it can be shown that the annular portion of the rod swept out by these swirling rays is given by $\left(n_{s} / n_{r}\right) r_{r o d}<r<r_{\text {rod. }}$. This means that if the gain depletion is severe, then only that portion of the rod lying inside a circular area with radius $\left(n_{s} / n_{r}\right) r_{\text {rod }}$ is useful for extracting laser energy from the rod as shown in Fig. 9. As an example, consider a YAG rod surrounded by cooling water, as is the case for our end-pumping technology under consideration here, then $\mathrm{n}_{\mathrm{r}}=1.82$ and $\mathrm{n}_{\mathrm{s}}=1.33$. In this case, if the swirling rays cause severe gain depletion, then only that portion of the rod contained within the central $53 \%\left(=100(1.33 / 1.82)^{2}\right)$ of the rod's cross sectional area would be useful for contributing to laser output power.
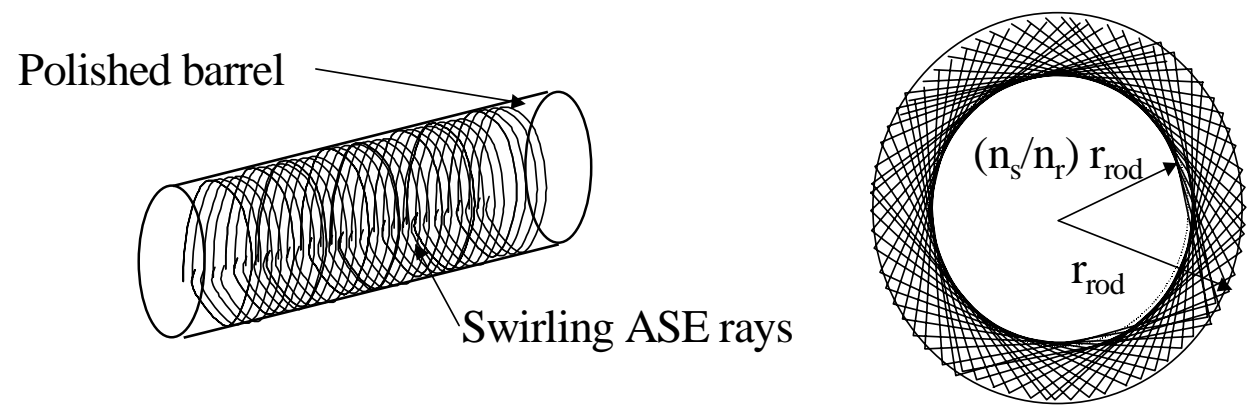

Fig. 9. Two different views of the trapped ray paths that can effectively deplete the stored laser gain in the annular region of the rod defined by $r_{\text {rod }}\left(n_{s} / n_{r}\right)<r<r_{\text {rod. }}$.

As already mentioned, the flanged endcap has been observed to be effective at decreasing the impact of ASE and parasitics on polished barrel laser rods. The flanged endcap works by limiting the maximum path length that can be trapped in the laser rod. To help visualize how this can be, first imagine a situation without a flanged endcap. Focussing on the straight polished barrel laser rod shown in Fig. 9, if the swirling rays of light wander to the end of the laser rod and then strike the end face of the rod at a high angle, they will be completely trapped at that face. In this situation the rays will be simply turned around and sent swirling down the rod in the other direction until they hit the output face at that end. This process is particularly bad from the viewpoint of depleting the stored laser energy in the rod, as these trapped rays now have an infinitely long trapped path length. However, using laser rods with flanged endcaps, once the swirling rays reach the end of the laser rod, the internal reflections made off the flanged part of the rod tend to straighten out the swirling ray, pointing it more along the axis of the laser rod. This in turn reduces the number of rays that will be confined by TIR on striking the end face of the rod and so effectively shortens the average path length of the trapped rays. In effect, one can think of the flanged endcap as acting like a sink for the swirling ASE rays, or as a "parasitic trap". In the lasers discussed in this manuscript, a symmetric rod design was used in which identical undoped endcaps are placed at both ends of the laser rods. Ideally, the flanged laser rod would limit the swirling rays described above to at most one pass down the length of the rod. However, even limiting the trapped ray paths to one pass can still allow for very long effective ray paths to be trapped, which in turn can pose a serious problem for gain 
depletion in the laser rod. These long trapped ray paths are characterized by swirling around many times while only moving a very short transverse distance up or down the laser rod. In fact, it is still possible for infinitely long ray paths to exist, which swirl around the laser rod but do not walk up or down its length.

One technique that we have developed and analyzed for limiting the maximum ray paths that can be trapped within the laser rod involves tapering the diameter of the laser rod over its length. In so doing, it can be shown that the maximum trapped path length of a barrel mode can be dramatically reduced, thereby reducing the ability of the trapped spontaneous emission to negatively impact laser performance through ASE. Figure 10 shows a sketch of a laser rod incorporating both undoped flanged endcaps and a tapered barrel. The rod barrel tapers in diameter from a small value of $d$ at one end to a larger value of $d+\varepsilon$ at the

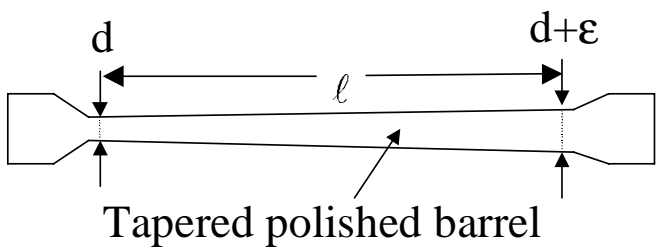

Fig. 10. Sketch of a tapered barrel rod used to eliminate long trapped path lengths.

other end. The impact of introducing a taper on the barrel of the laser rod is that it prevents swirling rays from loitering for extended path lengths in the laser rod. This is a significant improvement over the use of the flanged endcaps on a straight barreled rod alone, as the straight barrel rod configuration still allows trapped paths with infinite length. To estimate the maximum path lengths that can be trapped by a tapered laser rod with endcaps, an analysis based on a method of images construction has been developed. The reason for using the method of images construction in the analysis is that it simplifies the calculation of ray paths that involve TIR. Using the method of images, rather than having to calculate reflected angles and multiple bounce paths, rays are just straight-line propagated through reflective interfaces into image regions that are constructed from the original laser rod through the use of repeated reflections of the gain

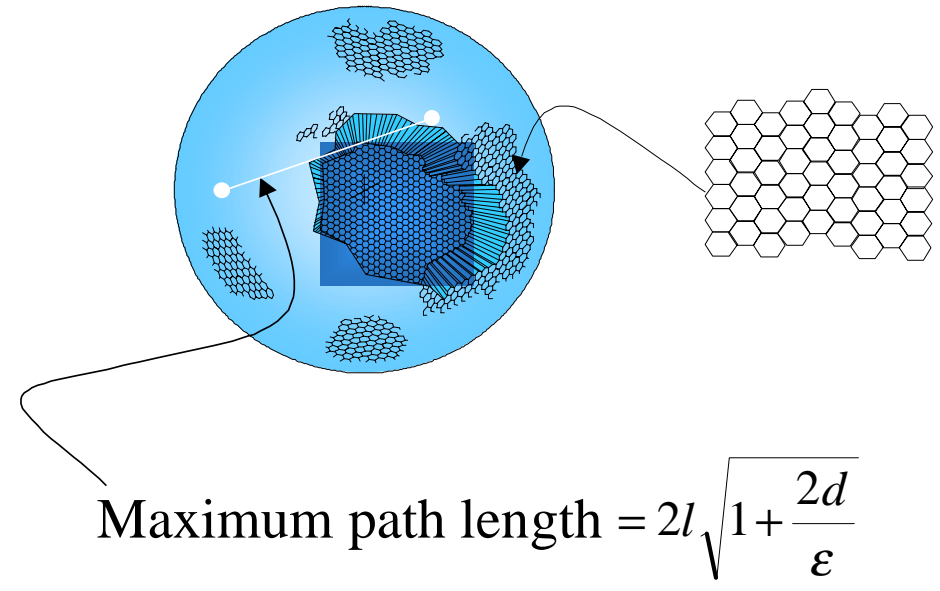

Fig. 11. Construction used to calculate the maximum trapped path length in a tapered barrel rod. The thickness of the spherical shell equals the length of the rod.

volume at the TIR surfaces. Of course, using this method does require that the constructed image space be contiguous in the sense of not containing any internal gaps. Unfortunately, round cross-sectioned rods do not meet the requirement of continuously filling a region of space on reflection about their various TIR surfaces. However, one simple approximation that allows the method of images to be applied to round rods models them as having an hexagonal cross-section. Additionally, this method assumes all rays that intercept the rod's surface are completely confined by reflection. Under these approximations, a rod with a taper generates a spherical shell with thickness equal to the length of the rod as displayed in Fig. 11. In this figure, the longest path length that is trapped in the laser rod is then the length of the longest line that can be drawn between any two points on the surface of the sphere. We are of course assuming that as the ray reaches the parasitic trap, it is completely defeated. 
In Fig. 11, a ray that intersects the surface of the sphere corresponds to a trapped ray, which has wandered into the flanged endcap at the rod end with the largest diameter. The longest trapped ray path is seen to correspond to a line that is tangent to the surface of the inner sphere. A simple calculation for the presently considered rod, with its diameter tapering from $d$ at one end to $d+\varepsilon$ at the other, then gives the length of this longest trapped path length as,

$$
\ell_{\max }=2 \ell \sqrt{1+\frac{2 d}{\varepsilon}}
$$

where $\ell$ is the length of the laser rod over which the taper occurs. Considering this expression for the longest trapped ray length, the question that naturally arises is what is the optimum value for the taper parameter $\varepsilon$. Clearly, if $\varepsilon$ approaches 0 , then the maximum path length neglecting diffraction effects can be pushed to infinity as has already been discussed for a straight rod. On the other hand, if $\varepsilon$ is made too large, then the useful mode volume of the rod is decreased. This last point is illustrated with the help of the Fig. 12.

In Fig. 12, the gray colored area corresponds to that portion of the rod that can be extracted with a laser beam in a conventional resonator. Due to the rod's taper, there is a portion of the laser rod's volume that

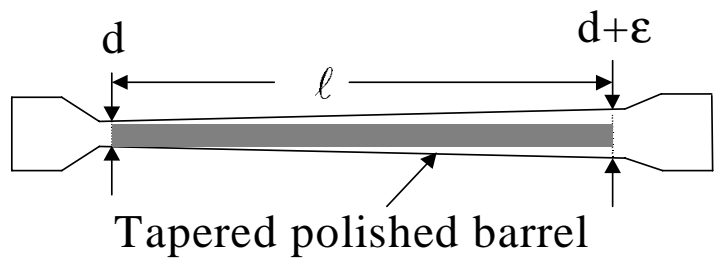

Fig. 12. The gray area in the sketch represents that portion of the rod volume from which energy can be extracted. The area outside the gray area is shadowed from the laser beam by the minimum diameter of the rod and so cannot be extracted.

will be inaccessible to an extracting laser beam due to the aperture introduced by the small end of the laser rod. Because this inaccessible volume grows as the taper grows, there will be an optimum taper value $\varepsilon$, which best balances the contradictory requirements of maximizing the size of the taper so as to minimize the longest trapped path length, and minimizing the size of the taper so as to maximize the fractional volume in the rod that is accessible to the extracting laser beam. The graphs in Fig. 13 show these two quantities plotted against the taper size for a $2 \mathrm{~mm}$ diameter by $5 \mathrm{~cm}$ long Yb:YAG laser rod, which is the
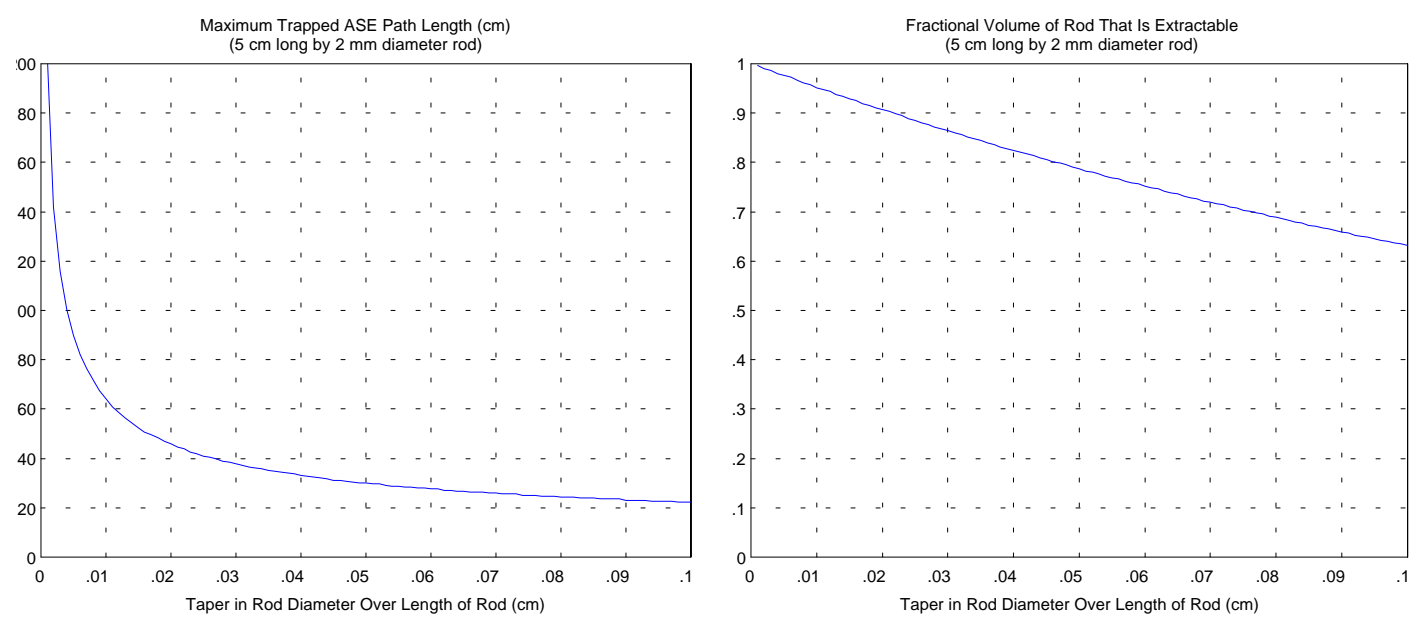

Fig. 13. The right graph plots the maximum trapped ASE path length as a function of the taper in the rod diameter for a rod that is $5 \mathrm{~cm}$ long and $2 \mathrm{~mm}$ in diameter at its narrowest point. The left graph plots the fractional volume of the rod that can be extracted as a function of the taper in the rod diameter. 
size used for our Yb:YAG experiments. Examining the plots in Fig. 13, it is apparent that there is very little to gain in terms of limiting the maximum ray path beyond taper values of $0.2 \mathrm{~mm}$, and at this taper value the rod volume that can be extracted is still 0.9.

A variation on the above approach that offers some performance benefit is shown in Fig. 14. Here, rather than having a continuous taper over the entire length of the laser rod, the center of the rod is the narrowest and the diameter increases as you go towards either end. The advantage of this geometry, with its dual taper, over a rod with a continuous taper from one end to the other, is that the maximum trapped path length in this rod is halved compared to that in the continuously tapered rod. Additionally, this halving of the maximum trapped path length comes without a penalty in the fractional volume of the rod from which energy can be extracted, assuming the same value of taper parameter, $\varepsilon$, for both types of rods. The rod design depicted in Fig. 14 is the design used in the lasers reported herein and have a doped section that is 5 $\mathrm{cm}$ long, and taper from a $2 \mathrm{~mm}$ diameter in their center to a $2.2 \mathrm{~mm}$ diameter at either end, and have undoped YAG rectangular flanged end caps that are $1.5 \mathrm{~cm}$ in length.

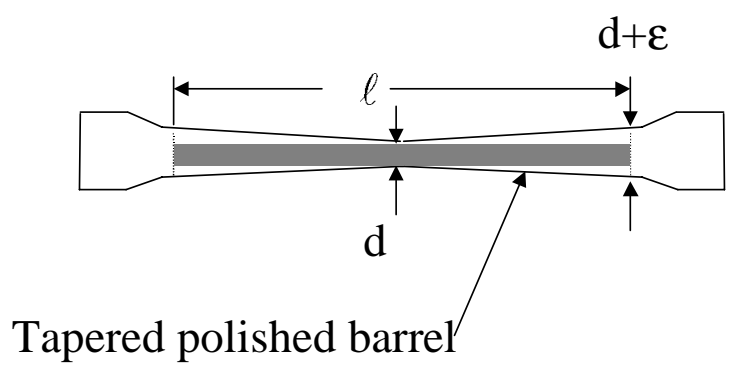

Fig. 14. Double tapered rod design used in our Yb:YAG laser.

\section{Dual rod Yb:YAG laser performance}

The approach that we have followed to achieve good beam quality at high-average power relies on designing our laser resonators to run with large diameter fundamental modes in the presence of strong thermal lensing ${ }^{1}$. With this approach, the laser rod itself acts as a spatial filter, limiting the number of transverse modes beyond the fundamental, which are supported by the cavity without incurring large diffractive losses. However, in single rod lasers such as the one shown in Fig. 1, stress-induced birefringence and bifocusing place a limit on the $\mathrm{TEM}_{0,0}$ mode size that can be produced ${ }^{15}$. In effect, what can happen is that the stability zones for the radially polarized light and the tangentially polarized light pull apart at high thermal loading due to the difference between the stress in the radial and tangential directions in the rod, leaving no possibility for stable mode operation of the laser cavity. One technique, which was originally demonstrated in 1970, can to a great extent eliminate birefringence and bifocussing effects by using two identically loaded rods separated by a $90^{\circ}$ rotator $^{16}$. Recently, there have been several reports of groups using this birefringence compensation technique to great success with diode-pumped Nd:YAG lasers $^{17}$. We have used the same approach to compensate for birefringence and bifocusing in our Yb:YAG laser. Figure 15 shows a schematic drawing and photograph of our dual-rod system with a $90^{\circ}$ rotator located in the center of the rods. The physical descriptions of the components used in the system are identical to the single-rod system. In particular, the same flanged and tapered laser rod design with a rectangular endcap and a $\mathrm{Yb}$ doping of $1 \times 10^{20} / \mathrm{cm}^{3}$ was used. Also, each of the two diode pump arrays used, one for each rod, have a capability of generating greater than $2 \mathrm{~kW}$ of $940 \mathrm{~nm}$ pump radiation.

Figure 16 plots the measured cw laser output power as a function of the applied diode pump power measured at the laser diode arrays. For pump powers greater than $3 \mathrm{~kW}$ it was found to be necessary to insert a negative lens between the two laser rods to maintain resonator stability. This negative lens was housed in the same fixture used to hold the $90^{\circ}$ rotator. The maximum output power achieved was $1080 \mathrm{~W}$ and was obtained with an input diode pump power (measured at the array) of $3930 \mathrm{~W}$ for an optical-optical efficiency of $27.5 \%$. At this pump power the electrical-to-optical conversion efficiency of the system was $12.3 \%$, although it is expected this value could be improved by reducing the number of diodes in the pump 
arrays as the diodes were running below their optimum efficiency point. The negative lens that was inserted in the resonator was varied as a function of the applied pump power and chosen so as to maintain a near constant $\mathrm{TEM}_{0,0}$ beam waist in the presence of the strong thermal lens in the laser rods. Because the $\mathrm{TEM}_{0,0}$ beam waist is held constant by the choice of compensating negative lens, it would be expected that the external beam quality of the developed laser radiation would also be approximately constant. Data that supports this assertion is displayed in Fig. 17, showing that the $\mathrm{M}^{2}$ of the beam is held approximately constant at 13.5 over the pump range that the compensating negative lenses were inserted in the cavity.
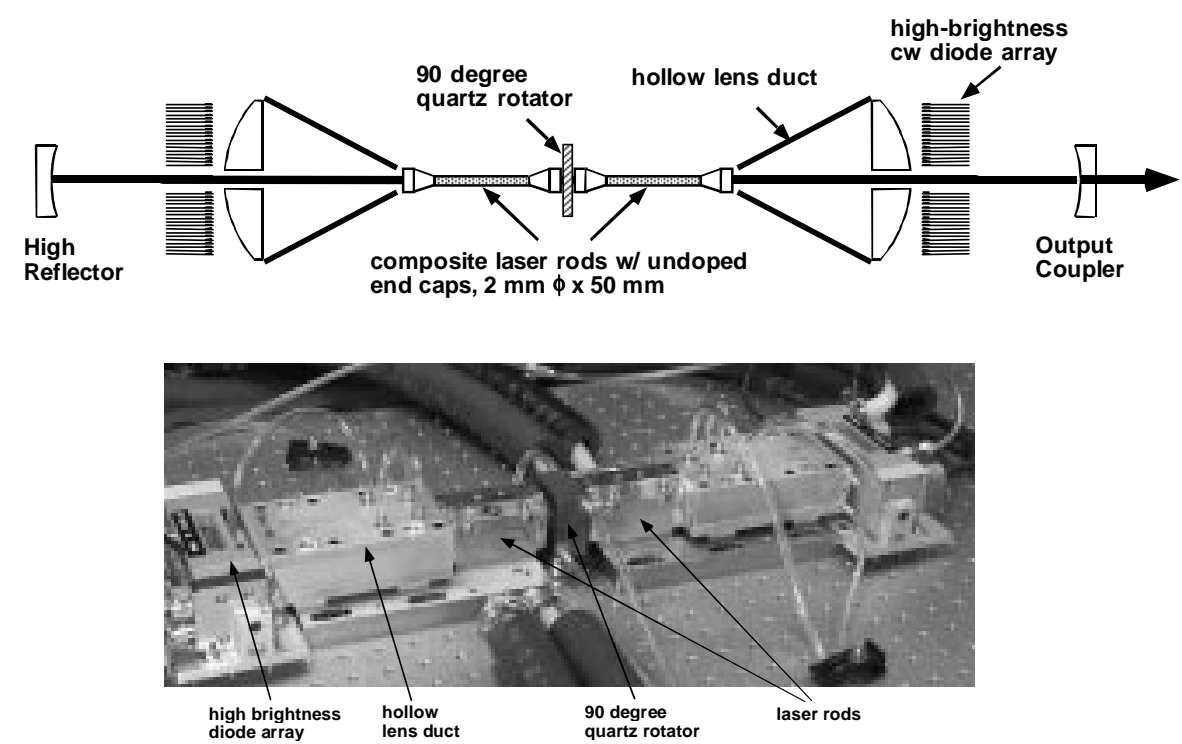

Fig. 15. Schematic diagram and photograph of our dual rod Yb:YAG laser with birefringence and bifocusing compensation

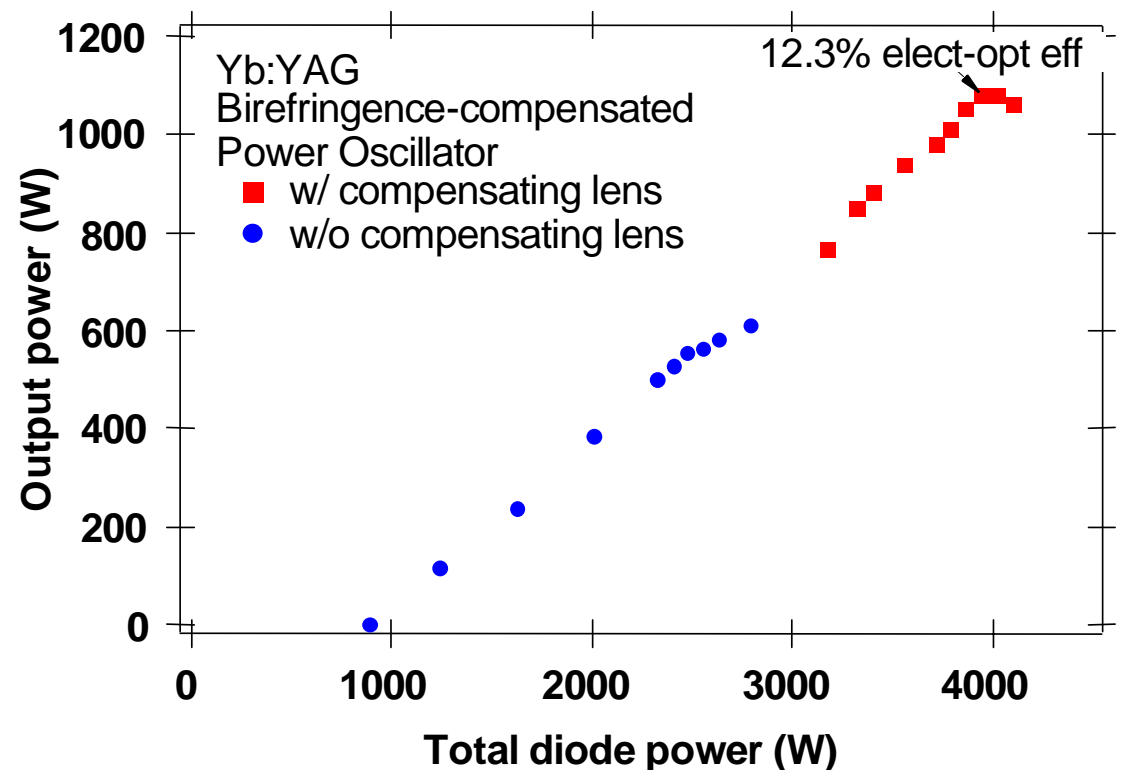

Fig. 16. Plot of measured cw output power from our birefringence and bifocusing compensated dual-rod Yb:YAG laser. At the high power end, cavity stability required the placement of a negative lens in the resonator between the two Yb:YAG laser rods. 


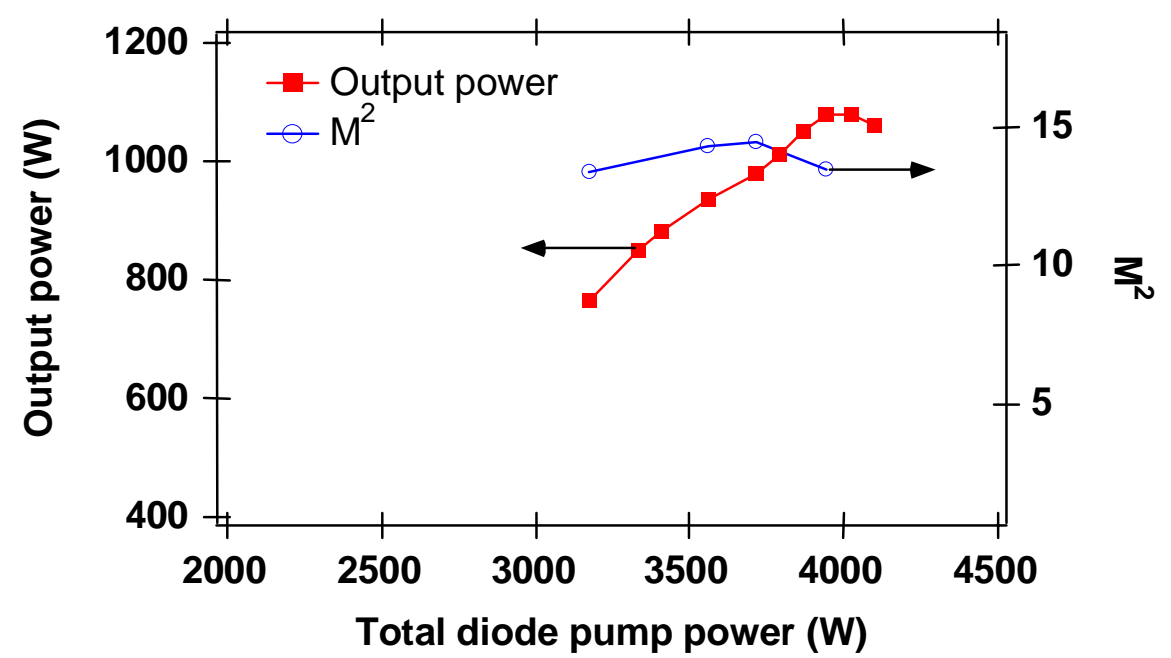

Fig. 17. At high cw powers, negative lenses were inserted in the resonator to maintain cavity stability. The focal strength of these lenses was varied with applied pump power to maintain a constant value $\mathrm{TEM}_{0,0}$ beam waist and so a near constant beam quality.

The calculations of the $\mathrm{TEM}_{0,0}$ beam waist are carried out using the Macintosh version of the software package Paraxia ${ }^{18}$. For each resonator configuration investigated, we use Paraxia to calculate the $\mathrm{TEM}_{0,0}$ beam waist expected in the laser rod, if it were running in a diffraction limited mode. Next using the embedded gaussian formalism ${ }^{19}$ applied to the intracavity laser beam, as discussed in reference [1], we can make a prediction as to the expected $\mathrm{M}^{2}$ value that the output laser beam will emerge from the cavity with. Essentially, we calculate the expected non-diffraction limited intracavity beam waist based on energetics modeling $^{20,21}$ and then get the $\mathrm{M}$ value by taking the ratio of the expected beam waist to the calculated $\mathrm{TEM}_{0,0}$ waist. Figure 18 plots the measured $\mathrm{M}^{2}$ for various negative lens values and resonator configurations along with our model predicted values.

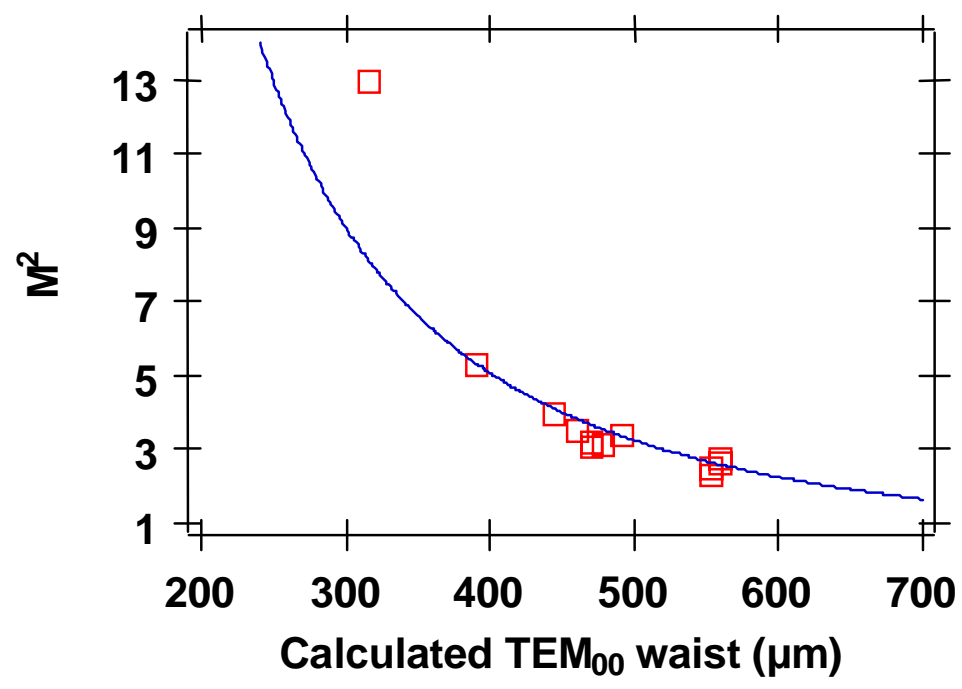

Fig. 18. Using the embedded gaussian model to predict beam quality $\left(\mathrm{M}^{2}\right)$ gave results in good agreement with our experimental measurements. 
In addition to evaluating the $\mathrm{cw}$ performance of the dual Yb:YAG rod laser system, we have performed initial experimental investigations of the q-switched performance of the system with the goal of generating output beams with $\mathrm{M}^{2}$ values less than 2.5. Q-switching was accomplished using two acousto-optic qswitches symmetrically located in the laser cavity between the laser diode arrays and the end mirrors. Under q-switched operation, the laser was $\mathrm{cw}$ pumped and q-switched at $10 \mathrm{kHz}$. A maximum q-switched average output power of $532 \mathrm{~W}$ was generated, corresponding to an optical-optical conversion efficiency of $17 \%$. Under these conditions, the generated pulse duration of the q-switched output pulses was $\sim 77 \mathrm{nsec}$, and the measured $\mathrm{M}^{2}$ of the beam was 2.3 in the horizontal direction and 2.0 in the vertical direction. $\mathrm{M}^{2}$ values were determined by calculating the second order moment of the output beam at various locations after a focussing lens and then fitting a hyperbolic curve as shown in Fig. 19.

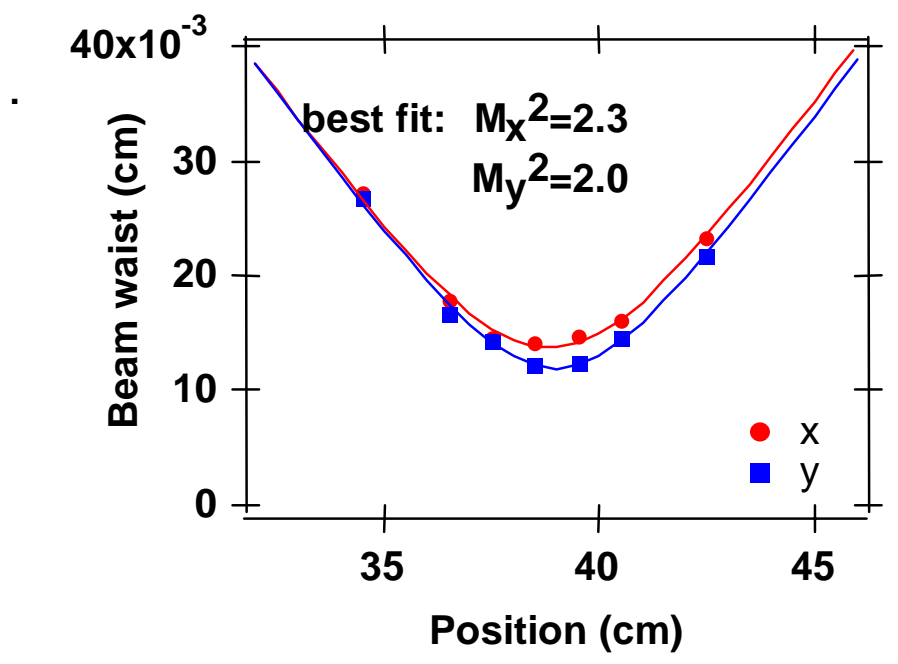

Fig. 19. Plot of second order moment in both the horizontal (x) and vertical (y) directions of the q-switched laser beam as a function of position after a focusing lens. The best-fit hyperbolic curves to this data yield the beam quality factors indicated.

\section{Summary}

We have continued to push the average power capability of our end-pumping technology and report here what we have accomplished with a Yb:YAG laser. With its greater than $80 \%$ demonstrated pump delivery efficiency, we believe our approach is the most efficient pump delivery technology that is available for delivering diode pump radiation into $2 \mathrm{~mm}$ diameter $\mathrm{Yb}$ :YAG rods. Additionally, the simplicity and robustness of the end-pumping technology make it attractive for a broad range of applications. Using a dual-rod configuration consisting of two $5 \mathrm{~cm}$ long by $2 \mathrm{~mm}$ diameter laser rods with birefringence and bifocusing compensation, we have achieved $1080 \mathrm{~W}$ of cw output with an $\mathrm{M}^{2}$ value of 13.5 at an optical-tooptical conversion efficiency of $27.5 \%$. With the same dual rod laser operated in a q-switched mode, we have demonstrated $532 \mathrm{~W}$ of average power with an $\mathrm{M}^{2}<2.5$ at $17 \%$ optical-to-optical conversion efficiency. These q-switched results were obtained at a $10 \mathrm{kHz}$ repetition rate and resulted in $77 \mathrm{nsec} \mathrm{q-}$ switched pulse durations. These improved levels of operational performance have been achieved through a number technology enhancements that include: (1) Hollow lens ducts that enable the use of advanced cavity architectures permitting birefringence compensation and the ability to run in large aperture-filling near-diffraction-limited modes. (2) Compound laser rods with flanged-nonabsorbing-endcaps fabricated by diffusion bonding. (3) Techniques for suppressing amplified spontaneous emission (ASE) and parasitics in the polished barrel rods. 


\section{Acknowledgments}

We wish to acknowledge many useful conversations with Bill Krupke, Rich Solarz, Luis Zapata and Howard Powell, all of LLNL, during the course of this work. We also acknowledge the expert laser technical assistance of Scott Mitchell and Joel Speth. We also thank Neil Ruggieri and Keith Sage of Boeing, for their help and many useful discussions during the course of this work. In addition, we thank Steve Mills, Barry Freitas, John Lang, Vic Sperry, Evert Utterback, Joe Santariano, Kathy Reinhardt, Larain Dimercurio, and Sally Gonzales all of LLNL in carrying out various portions of the work reported. We are indebted to Helmuth Meissner and Oliver Meissner of ONYX Optics for fabricating the composite laser rods with diffusion bonded end-caps, Ralph Hucheson of Scientific Materials for growing the YAG crystals used in our lasers, and Karl George of Quality Thin Films for supplying the optical coatings used on our laser rods. This research was performed under the auspices of the U.S. Department of Energy by Lawrence Livermore National Laboratory under contract W-7405-ENG-48.

${ }^{1}$ E.C. Honea, R.J. Beach, S.C. Mitchell, and P.V. Avizonis, "183-W, $\mathrm{M}^{2}=2.4$ Yb:YAG Q-switched laser," Opt. Lett. 24, p. 154, 1999.

${ }^{2}$ E. C. Honea, R.J. Beach, S.C. Mitchell, and P.V. Avizonis, "183 W, M²=2.4 Yb:YAG Q-switched laser," CLEO '99 in Baltimore, MD, Technical Digest Series, talk CMF2.

${ }^{3}$ R.J. Beach, C. Bibeau, E.C. Honea, S.B. Sutton, "Taking Average-Power, Diode-Pumped, Solid-State Lasers Beyond the $\mathrm{Nd}^{3+}$ Ion," Inertial Confinement Fusion 1997 ICF Annual Report, Lawrence Livermore National Laboratory, J. Carpenter and D. Correll (eds.) UCRL-LR-105821-97-2, pp. 52- 62, 1997.

${ }^{4}$ D.S. Sumida, A.A. Betin, H. Brusselbach, R. Byron, S. Matthews, R. Reeder, and M.S. Mangir, "Diodepumped Yb:YAG catches up with Nd:YAG,” Laser Focus World, pp. 63-70, June 1999.

${ }^{5}$ R. Beach, P. Reichert, W. Benett, B. Freitas, S. Mitchell, S. Velsko, J. Davin, and R. Solarz, "Scalable diode-end-pumping technology applied to a 100-mJ Q-switched $\mathrm{Nd}^{3+}$ :YLF laser oscillator," Opt. Lett., vol. 18, pp. 1326-1328, 1993.

${ }^{6}$ R. Beach, W. J. Bennett, B. L. Freitas, D. Mundinger, B. J. Comaskey, R. W. Solarz and M. A. Emanuel, "Modular microchannel cooled heatsinks for high average power laser diode arrays," IEEE J. Quant. Elect., vol. 28, pp. 966-976, 1992.

7 J. Snyder, P. Reichert, and T. Baer, "Fast diffraction limited cylindrical microlenses," Appl. Opt., vol. 30, pp. 2743-2747, 1991.

${ }^{8}$ R. Beach, M.A. Emanuel, B.L. Freitas, J.A. Skidmore, N.W. Carlson, W.J. Benett, and R.W. Solarz, “Applications of microlens-conditioned laser diode arrays," SPIE Vol. 2383, pp. 283-297, 1995.

${ }^{9}$ R.J. Beach, “Theory and optimization of lens ducts,” Appl. Opt., vol. 35, pp. 2005-2015, 1996.

${ }^{10}$ E.C. Honea, R.J.Beach, S.B. Sutton, J.A. Speth, S.C. Mitchell, J.A. Skidmore, M.A. Emanuel, and S.A. Payne, “115-W Tm:YAG Diode-Pumped Solid-State Laser,” IEEE J. Quant. Elect., vol. 33, pp. 1592-1600, 1997.

${ }^{11}$ C.B. Bibeau, R.J. Beach, S.C. Mitchell, M.A. Emanuel, J. Skidmore, C.A. Ebbers, S.B. Sutton, and K.S. Jancaitis, "High-Average-Power 1- $\mu \mathrm{m}$ Performance and Frequency Conversion of a Diode-End-Pumped Yb:YAG Laser,” IEEE J. Quant. Elect., vol. 34, pp. 2010-2019, 1998.

${ }^{12}$ R.J. Beach, S.B. Sutton, E.C. Honea, J.A. Skidmore, and M.A. Emanuel, "High power $2 \mu \mathrm{m}$ wingpumped $\mathrm{Tm}^{3+}$ :YAG laser," OSA TOPS on Advanced Solid-State Lasers, 1996 Vol. 1, S.A. Payne and C. Pollock (eds.), pp. 213-215, 1996. 
${ }^{13}$ ONYX Optics Corporation,6551 Sierra Lane, Dublin, CA 94568

${ }^{14}$ J. Linn and J. Free, "Effect of Trapped Light on the Output of a Ruby laser," Applied Optics, 4, p 1099, 1965.

${ }^{15}$ M.P. Murdough and C.A. Denman, "Mode-volume and pump-power limitations in injection-locked TEM(00) Nd:YAG rod lasers," Applied Optics 35 pp. 5925-5936, 1996.

${ }^{16}$ W.C. Scott and M. de Wit, "Birefringence Compensation and TEM $_{00}$ Mode Enhancement in a Nd:YAG Laser," App. Phys. Lett. 18, pp.3-4, 1971.

${ }^{17}$ S Fujikawa, S. Konno, K. Yasui, and K. Yoshizawa, "Highly efficient diode-pumped 300-W Nd:YAG rod laser," OSA TOPS Vol. 26 Advanced Solid-State Lasers, M.M. Fejer, H.I. Injeyan, and U. Keller (eds.), pp. 5-8, 1999.

${ }^{18}$ Paraxia software from Sciopt Enterprises, San Jose California.

${ }^{19}$ A.E. Siegman, Proc. SPIE 1224, 2 (1990).

${ }^{20}$ R.J. Beach, "CW Theory of quasi-three level end-pumped laser oscillators," Opt. Comm. 123, pp. 385393, 1996.

${ }^{21}$ R.J. Beach, "Optimization of Quasi-Three level End-Pumped Q-Switched Lasers," IEEE J. Quant. Elec. 31, pp. 1606-1613, 1995. 